\title{
A Practical Guide to Analyzing Time-Varying Associations between Physical Activity and Affect Using Multilevel Modeling
}

\author{
Jinhyuk Kim (D), ${ }^{1}$ David Marcusson-Clavertz, ${ }^{1,2}$ Fumiharu Togo, ${ }^{3}$ and Hyuntae Park ${ }^{4}{ }^{4}$ \\ ${ }^{1}$ Department of Biobehavioral Health, The Pennsylvania State University, University Park, PA, USA \\ ${ }^{2}$ Department of Psychology, Lund University, Lund, Sweden \\ ${ }^{3}$ Educational Physiology Laboratory, Graduate School of Education, The University of Tokyo, Tokyo, Japan \\ ${ }^{4}$ Department of Health Care and Science, College of Health Science, Dong-A University, Busan, Republic of Korea
}

Correspondence should be addressed to Jinhyuk Kim; juk423@psu.edu and Hyuntae Park; htpark@dau.ac.kr

Received 14 December 2017; Revised 15 May 2018; Accepted 24 May 2018; Published 9 July 2018

Academic Editor: Zoran Bursac

Copyright (c) 2018 Jinhyuk Kim et al. This is an open access article distributed under the Creative Commons Attribution License, which permits unrestricted use, distribution, and reproduction in any medium, provided the original work is properly cited.

\begin{abstract}
There is growing interest in within-person associations of objectively measured physical and physiological variables with psychological states in daily life. Here we provide a practical guide with SAS code of multilevel modeling for analyzing physical activity data obtained by accelerometer and self-report data from intensive and repeated measures using ecological momentary assessments (EMA). We review previous applications of EMA in research and clinical settings and the analytical tools that are useful for EMA research. We exemplify the analyses of EMA data with cases on physical activity data and affect and discuss the future challenges in the field.
\end{abstract}

\section{Introduction}

Enabled by technological developments, ecological momentary assessment (EMA) [1] using mobile data collection has become an essential research tool in many fields of social and behavioral sciences and is continuing to spread to other areas of sciences. EMA research covers a wide range of phenomena, including the study of environmental, physical, physiological, psychological, and sociological factors, using repeated or continuous recording. Given the widespread use of EMA methods across the different sciences, various terms have been used to refer to similar procedures. EMA methods focusing on self-report data are frequently referred to as experience-sampling methods (ESM) [2], whereas those focusing on physical, physiological, or biological data are often called ambulatory assessments (AA) [3]. However, we use the term EMA broadly to include all these types of ecological, intensive assessments. To exemplify, EMA studies investigate various behaviors, experiences, and environmental conditions, including depression [4-6], psychological stress $[7,8]$, self-esteem [9], diet [10], self-reported physical activity [11, 12], smoking [13-15], sexual behavior [16], compulsive buying [17], social interaction [4, 18], work activity and satisfaction $[8,19]$, diabetes management $[18$, $20]$, effects of medication [21, 22], asthma [23, 24], allergies $[25,26]$, tinnitus [27], and working memory and attention [28]. In addition, technological developments have enabled automated EMA of behaviors (e.g., taking medication [29]) and physical environment (e.g., air sampling [26], sampling in electromagnetic fields [30]). Ambulatory monitoring of cardiovascular function, using portable cardiac monitors, has been used for several decades as a tool for understanding the association between experiences and cardiovascular health [31]. Recent developments have expanded physiological monitoring to other parameters, such as physical activity [32-38], hypothalamic-pituitary-adrenal axis activity [39-42], blood glucose [43], skin temperature [44], pulmonary function [23], and others. Furthermore, these data collections are widely used to evaluate treatment and intervention of crucial health-related behaviors in health psychology and behavioral medicine, such as coping with illness and treatment [45, $46]$, medication compliance $[47,48]$, and exercise $[49,50]$. 
Psychiatric (or psychosomatic) disorders studied with EMA include a wide range of psychopathology, such as addictive disorders [51, 52], gastrointestinal disorders [53], sexual dysfunction [54], eating disorders $[46,55,56]$, attention deficit hyperactivity disorder (ADHD) [57, 58], mood dysregulation [59], anxiety disorders [60-62], depressive disorders [63-65], bipolar disorder [66], and schizophrenia [67-69].

Why did EMA become frequently used in various areas of researches including clinical settings? One advantage of EMA methods is that they enable us to study a phenomenon in its natural environment. A second advantage is that they allow us to study the time course of target variables. Intensive data collection enables the exploration of development trajectories of psychiatric disorders and physical health conditions and identification of factors that are predictive of these trajectories. For instance, one study used EMA methods to examine if spousal responsiveness to verbal expressions of pain in patients with knee osteoarthritis predicted patients' physical function over time [70]. Such fluctuations in the trajectories cannot be captured by traditional, cross-sectional data collection methods. A third advantage is that EMA enable us to assess related symptoms with other related factors (e.g., physiological states or social and environmental situations) immediately before and just after disorders (e.g., panic attacks [62], binge eating [55]), which give us important insights into pathogenic processes and prevention of psychiatric disorders and poor physical health.

Many EMA studies have examined how a phenomenon covaries with variables that may vary across different levels, including moments (e.g., mood states), days (e.g., work days versus weekends), persons (e.g., unemployed versus employed), or other levels (e.g., organizations, seasons) in various populations, including patients with psychiatric disorders and physical conditions. For example, cardiovascular reactivity [71-74] and cortisol-related reactivity [39-42, 75] were reported to be associated with levels of psychological stress, and changes in pulmonary functions tested by a spirometer were associated with daily positive/negative affect, as well as the symptom of shortness of breath in asthma patients. Health-related behaviors, such as eating [76, 77], smoking [13, 14], and alcohol consumption [78, 79], exhibited associations with variation in physical symptoms and psychological states, e.g., craving, positive/negative affect, and anxiety. Furthermore, associations between physical activity measured by self-report and daily fluctuations in psychological states have been reported $[11,12]$. These studies provide strong evidence that biological/physiological measures vary in time with momentary symptoms. Thus, the existence of such objective proxies for subjective symptoms indicates the possibility of the practical use of them for monitoring momentary symptoms in a continuous fashion (i.e., without the need for self-reports). There might also be advantages in simultaneously using self-reported subjective symptoms and objective measures to improve the explanation of health outcomes.

It has been suggested that momentary fluctuations in behavioral data, specifically those on physical activity capturing bodily acceleration, reflect the dynamics of systems organizing human behavior and can be used to examine behavioral disorders, including mental illnesses [32-38]. Indeed, altered physical activity is one of the cardinal signs of psychiatric disorders and included in their diagnostic criteria [80]. For example, major depressive disorders (MDD) are characterized by the presence of symptoms associated with behavioral alterations, including diminished physical activity, psychomotor retardation or agitation, and sleep disturbances [80]. Specifically, several studies using accelerometer have been conducted with patients with depression, showing disruption of the circadian rhythm [32-34]. Research has shown the existence of robust statistical regularities concerning daily life behaviors, specifically how resting and active periods derived from physical activity data are interwoven into daily life [81]. In addition, this research found, in patients with $\mathrm{MDD}$, a significant alteration of a parameter of the robust law representing the distribution of resting periods; compared to healthy subject, these patients exhibited more intermittent behavioral patterns characterized by reduced mean activity levels associated with occasional bursts of physical activity counts [81, 82]. Furthermore, alterations of intermittent properties of physical activity have been reported in schizophrenia and bipolar disorder [83, 84]. Recent studies showed the psychobehavioral correlates of temporal diurnal fluctuations in momentary depressive mood and behavioral dynamics $[85,86]$. The results in these studies suggested that an increased intermittency of physical activity (i.e., low mean level and occasional burst of physical activity) appeared concurrently with the worsening of depressive mood in healthy subjects across a wide range of populations (adolescents, undergraduates, and adult office workers) [85], as well as in patients with MDD [86]. Furthermore, the cross validation between healthy subjects and patients with MDD were confirmed, indicating that the same psychobehavioral correlates are shared by both groups [86]. A pilot study suggested that temporal variations in depressive mood are affected by underlying changes in physical activity in older adults. Reduced activity patterns preceded or occurred concurrently with the worsening of depressive mood rather than following (Figures 1(a) and 1(b)) [87]. These findings suggest that physical activity obtained by accelerometer is a useful measure for evaluating behavioral abnormalities associated with psychiatric disorders, and that its characterization is likely to provide an objective measure for these disorders. However, other studies have not found support for associations between some types of mental disorders or psychological states and physical activity. For example, a study reported nonsignificant bidirectional associations between mood (i.e., energetic arousal, valence, and calmness) and physical activity in inactive university students [88]. Another study showed that physical activity contributes to an improvement of positive affect, but not a reduction of negative affect in MDD [89].

In this paper, we describe analytic models that are useful for analyzing EMA data with cases on physical activity data and affect. We also offer Supplementary Materials (available here) with SAS code for how to handle physical activity data obtained by accelerometer and use multilevel modeling techniques on EMA data. 


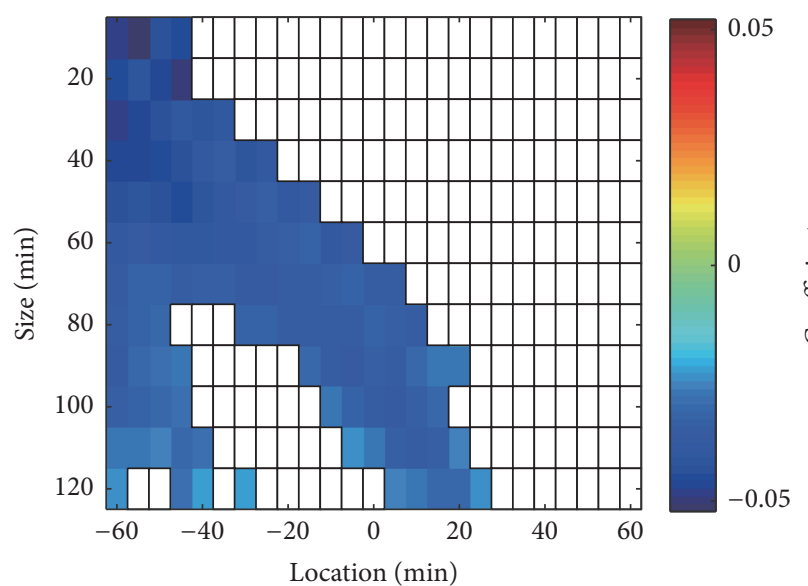

(a) Mean

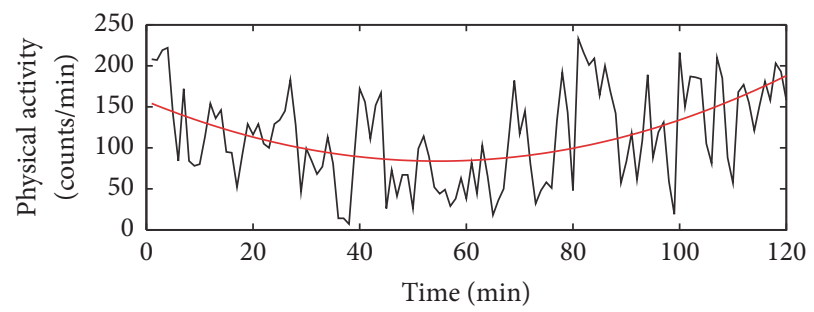

- Raw physical activity

— Second-order polynomial

(c) Raw physical activity

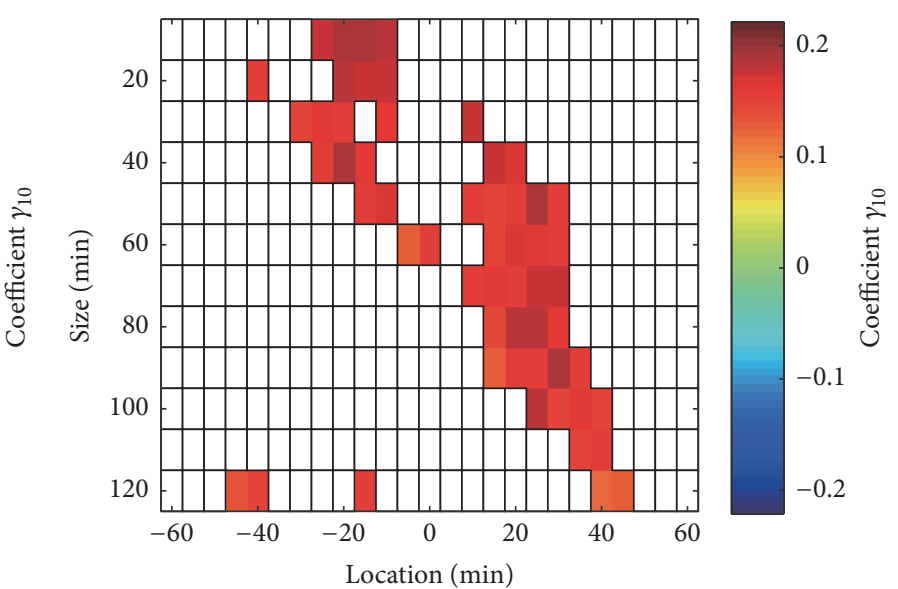

(b) Detrended skewness

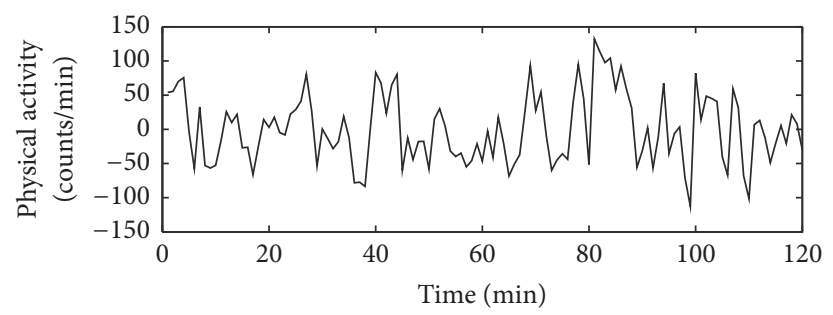

(d) Detrended physical activity

Figure 1: Analytic techniques for physical activity. (a) The temporal associations of depressive mood and local mean of physical activity which evaluates lower/higher mean activity levels. Estimated values of the univariate multilevel model coefficient for the associations are shown in a colored matrix form consisting of 25 columns (different location) and 12 rows (different size) in older adults $(n=9)$. Each grid cell indicates specific location and size of a time frame used for calculating the local mean of physical activity surrounding each EMA recording of depressive mood. A color in each cell represents the value of the model coefficient $\left(\gamma_{10}\right)$ of the predictors. The false discovery rate with the $q$ value of .05 was used as the multiple comparison adjustment. Only the significant cases were shown by colors. Note that the univariate model used for the analysis is as follows. Depressive mood score $t_{j}=\gamma_{00}+\gamma_{10}$ (local statistics of physical activity $\left.{ }_{t j}\right)+\zeta_{0 j}+\varepsilon_{t j}$ [see [85] for details]. (b) The same is shown in panels (a), except for the local mean. Local skewness of physical activity, which evaluates asymmetry of a distribution (i.e., occasional bursts of physical activity in a time window), was used in this panel. (c) A raw physical activity time series for 120 min and the second-order polynomial line (red). (d) The detrended physical activity derived by subtracting the fitted line for the original data.

\section{Analytic Tools and Techniques for Evaluating Time-Varying Associations between Physical Activity and Affect}

2.1. Multilevel Modeling. Although there are several analytical approaches to examine the association between physical activity and affect in daily life (e.g., correlation, regression, or time series modeling), multilevel modeling is suitable for addressing unbalanced and hierarchical EMA data. In EMA data, multiple observations are typically hierarchically nested within individuals, with the number and timing of observations varying between individuals (see Figure 2 for an example of EMA data structure). In addition, EMA studies usually have missing data due to difficulties in fully complying with the schedule. Traditional techniques such as repeated measures analysis of variance (RM-ANOVA) are not suitable for analyzing these unbalanced data sets [90]. However, such data can be handled by the multilevel modeling approach, which is an extension of traditional regression models and has been recommended for the analysis of data with a hierarchical structure (Figure 2) [90-92].

In multilevel modeling, these within- and betweenindividual effects can be handled together in the same model by incorporating random effects into model coefficients, i.e., allowing the coefficients to vary across individuals. For example, a researcher might expect that the average level of physical activity and the influence of negative affect on physical activity differ significantly between individuals, and therefore, model these effects as random intercepts and slopes, respectively. Although the multilevel model can be expressed as a single equation, it is easier to understand if it is initially presented as a set of equations separating withinand between-individual levels. In EMA analysis, usually observations are modeled as level 1 (within-individual level) 
Hierarchical data structure

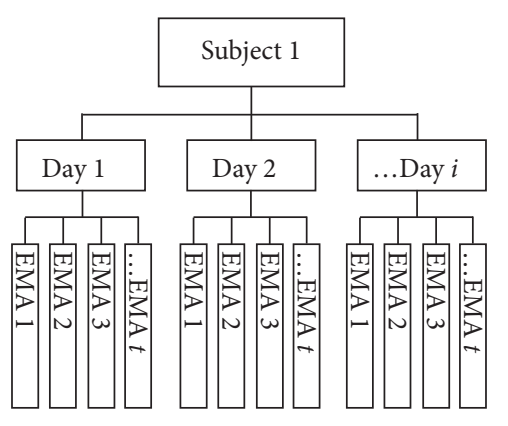

Single-level regression model

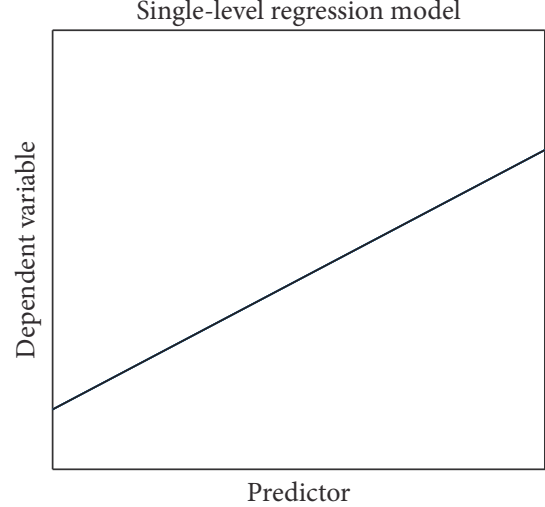

(b)

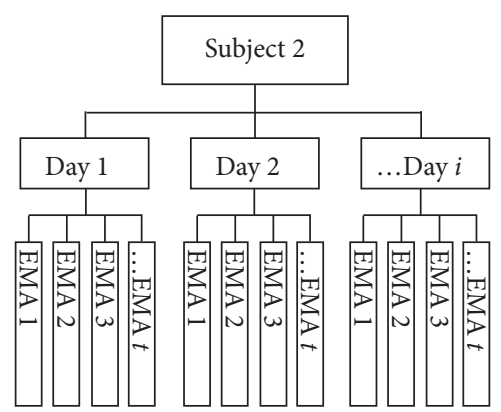

(a)

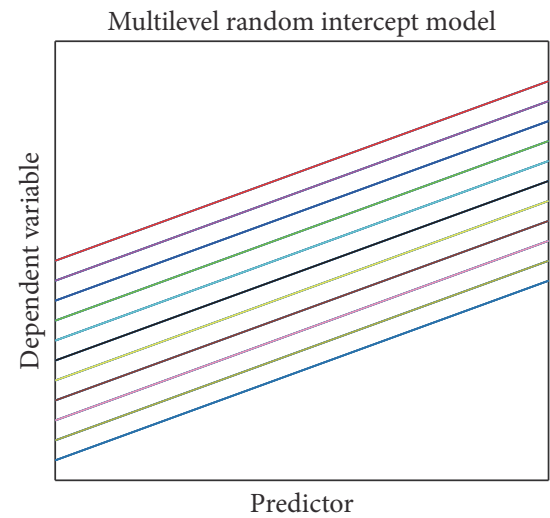

(c)
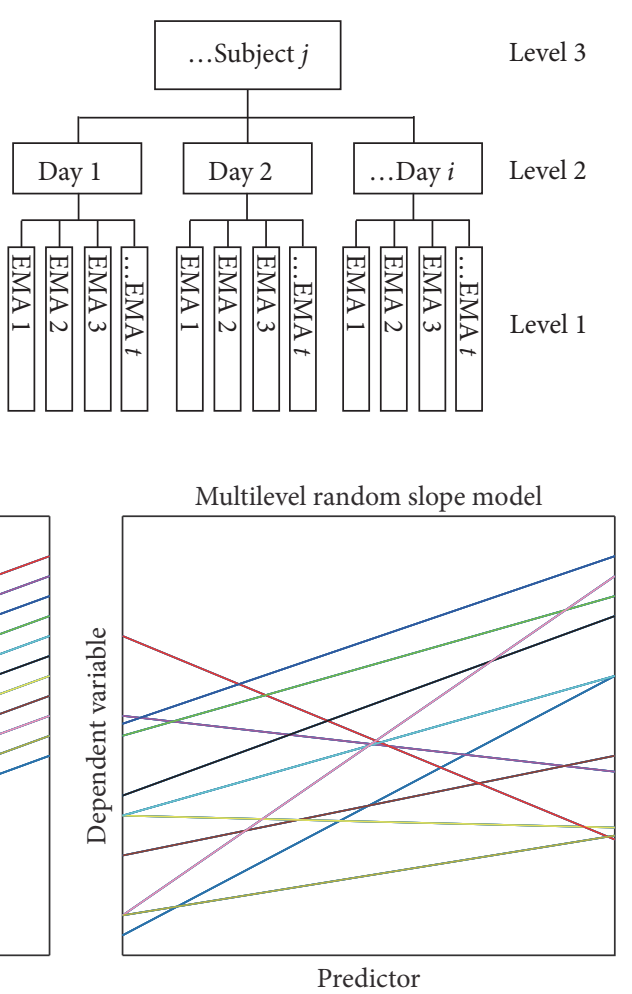

(d)

FIGURE 2: Concept plots illustrate multilevel modeling using hierarchical ecological momentary assessment (EMA) data. (a) An example of hierarchical data structure in which EMA observations (level 1) nested within days (level 2) nested within subjects (level 3). The number of EMA observations $(t)$ and days (i) can be different in each subject. (b) Traditional regression model with fixed slope and intercept which do not vary across subjects. (c) Multilevel model with random intercepts, which vary across subjects, and fixed slopes. (d) Multilevel model with random intercepts and slopes. The multilevel model can be tested with random slopes and fixed intercepts, but the practical use of the model may be limited.

units nested within individuals who are modeled as level 2 (between-individuals level) units. An example of multilevel models is as follows.

Level 1 Equation (Within-Individual [Observation] Level)

$$
Y_{t j}=\pi_{0 j}+\sum_{k=1}^{n} \pi_{k j}\left(X_{t j}^{k}-\bar{X}_{j}^{k}\right)+\varepsilon_{t j} \quad(k=1, \ldots, n)
$$

where $Y_{t j}$ indicates the dependent variable (e.g., negative affect or depression) $t$ th momentary observation for the $j$ th subject; $X_{t j}^{k}$ is the $k$ th predictor (e.g., physical activity; $k$ represents the order of predictors) corresponding to the $t$ th momentary observation for the $j$ th subject; $\bar{X}_{j}^{k}$ is the person mean of the $k$ th predictor for centering to estimate the within-person effect of the predictor (physical activity) on the dependent variable of subjective symptoms [93]; $n$ is the total number of predictors; $\pi_{0 j}$ and $\pi_{k j}$ are the subject $j$ 's intercept and coefficient (i.e., slope) of the predictor, respectively; and $\varepsilon_{t j}$ is the within-individual residual.

\section{Level 2 Equations (Between-Individual Level)}

$$
\begin{aligned}
& \pi_{0 j}=\gamma_{00}+\gamma_{01} Z_{j}+\zeta_{0 j} \\
& \pi_{k j}=\gamma_{k 0}+\gamma_{k 1} Z_{j}+\zeta_{k j}
\end{aligned}
$$

where $\gamma_{00}$ is the average intercept across all subjects; $\gamma_{k 0}$ is the average slope across all subjects; $Z_{j}$ is the between-individual level predictor representing, e.g., subject's characteristics; $\gamma_{01}$ and $\gamma_{k 1}$ are the effect of the variable $Z_{j}$; and the random terms $\zeta_{0 j}$ and $\zeta_{k j}$ are the between-individual residuals.

\section{Combined Model}

$$
\begin{aligned}
Y_{t j}= & \gamma_{00}+\gamma_{01} Z_{j}+\sum_{k=1}^{n} \gamma_{k 0}\left(X_{t j}^{k}-\bar{X}_{j}^{k}\right) \\
& +\sum_{k=1}^{n} \gamma_{k 1} Z_{j}\left(X_{t j}^{k}-\bar{X}_{j}^{k}\right)+\zeta_{0 j}+\sum_{k=1}^{n} \zeta_{k j}\left(X_{t j}^{k}-\bar{X}_{j}^{k}\right) \\
& +\varepsilon_{t j}
\end{aligned}
$$

When the groups are nested within additional groups, the data form a 3-level hierarchy and 3-level models can be fitted 
to account for the additional level, e.g., EMA observations (level 1) nested within days (level 2) nested within individuals (level 3). An example of 3-level multilevel models is as follows (combined model is not shown).

Level 1 Equation (Within-Individual [Observation] Level)

$$
Y_{t i j}=\pi_{0 i j}+\sum_{k=1}^{n} \pi_{k i j}\left(X_{t i j}^{k}-\bar{X}_{j}^{k}\right)+\varepsilon_{t i j} \quad(k=1, \ldots, n)
$$

where $Y_{t i j}$ indicates the dependent variable at the $t$ th momentary observation for the $j$ th subject on the $i$ th day; $X_{t i j}^{k}$ is the $k$ th predictor corresponding to the $t$ th momentary observations for the $j$ th subject on the $i$ th day; $\pi_{0 i j}$ and $\pi_{k i j}$ are the subject $j$ 's intercept and coefficient (i.e., slope) of the predictor on the $i$ th day, respectively; and $\varepsilon_{t i j}$ is the observation-level residual.

Level 2 Equations (Within-Individual [Day] Level)

$$
\begin{aligned}
& \pi_{0 i j}=\beta_{00 j}+\zeta_{0 i j} \\
& \pi_{k i j}=\beta_{k 0 j}+\zeta_{k i j}
\end{aligned}
$$

where $\beta_{00 j}$ is the subject $j$ 's intercept. $\beta_{k 0 j}$ is the subject $j$ 's slope; and the random terms $\zeta_{0 i j}$ and $\zeta_{k i j}$ are the day-level residuals.

\section{Level 3 Equations (Between-Individual Level)}

$$
\begin{aligned}
& \beta_{00 j}=\gamma_{000}+\gamma_{001} Z_{j}+\delta_{00 j} \\
& \beta_{k 0 j}=\gamma_{k 00}+\gamma_{k 01} Z_{j}+\delta_{k 0 j}
\end{aligned}
$$

where $\gamma_{000}$ and $\gamma_{k 00}$ are the average intercept and slope across all subjects, respectively; $Z_{j}$ is the between-individual level predictor representing, e.g., subject's characteristics; $\gamma_{001}$ and $\gamma_{k 01}$ are the effect of the variable $Z_{j}$; and the random terms $\delta_{00 j}$ and $\delta_{k 0 j}$ represent the residuals on the betweenindividual level. See SAS codes in the Supplementary Materials (available here) for the above models.

\subsection{Which Statistics Should Be Used to Characterize Physical} Activity? Accelerometer is commonly used to objectively measure physical activity and capable of detecting large volumes of small changes in bodily acceleration. A common accelerometer method is to count zero-crossing activities; that is, the number of times that the signal crosses zero within the buffer [94], accumulated to 1-min epochs (we will assume this method in the discussion below, but there are also other ways to assess accelerometer data). The accelerometer enables opportunities to improve the characterization of activity patterns in daily life but also brings new analytic challenges despite expanding efforts to address these issues [95]. A study examined several issues with the use of accelerometer data on algorithms for the time of wearing or taking off the device and activity cut-off points for different intensities of physical activity [96]. The study showed that the choice of epoch length, which refers to the interval of time over which the units of accelerometer measures are aggregated (e.g., 15 seconds or 1 minute), may introduce significant errors when the chosen epoch length mismatches the length originally used for validating the wear time algorithm and activity cutoff points. This indicates that wear time or time spent in different intensities of physical activity cannot be directly compared across studies unless they used the same epoch lengths [96].

In addition to characterizing general activity patterns in daily life, accelerometers are useful tools for estimating the extent of a person's movement over a given period of time, including the intensity, duration, frequency, and the type of movement [95]. There has been growth in research on time spent in different intensities of physical activity (e.g., sedentary behavior [97-100], light, moderate, and vigorous physical activity [101-103]), but a common accelerometer measure is the activity counts per a certain period of time, which represent total volume of physical activity.

Although there are several important issues to consider when analyzing accelerometer data, we focus on how to characterize local (i.e., temporal) physical activity patterns surrounding EMA recordings of affect. To extract and characterize activity patterns in a temporal time window, researchers can analyze local statistics of physical activity data up to the fourth-order moment (i.e., mean, standard deviation [SD], skewness, and kurtosis) around EMA recordings (e.g., 60min local mean of physical activity around the EMA signal). However, a research group focused on mean and skewness because they considered first- and third-order moments to be sufficient to characterize the observed accelerometer data $[85,86]$. While $S D$ (i.e., the second-order moment) is a standard measure characterizing variability of data, it can be inappropriate when the data do not approximate a normal distribution; the distribution of physical activity has nonnegative values, leading to a positively skewed distribution. Intermittency or non-Gaussianity in natural phenomena is known to be successfully captured by the higher-order statistics, such as nonzero skewness or larger kurtosis (flatness) of the probability distribution of the observed data [104, 105], corresponding to the presence of frequent bursts. Indeed, the local SD of physical activity did not play a major role in predicting affect (i.e., depressive mood) scores $[85,86]$. In contrast, the skewness, as a measure of asymmetry of a distribution, is thought to be more appropriate to characterize the observed asymmetry. Lower or higher mean activity levels quantify the overall states of physical activity. Higher positive skewness quantifies occasional bursts of physical activity [8183]. Other local statistics of physical activity that can capture the intermittency in physical activity more robustly, such as entropy-type nonlinear statistics, can also be considered.

It is important to consider the effect of time of day on physical activity. A simple way to address this would be by adding a term for time of day (e.g., every 4 hours or morning/afternoon/evening blocks) to (1) or (5) as a controller or moderator [106]. We can also use detrended activity data [85], where a diurnal trend of activity data is subtracted by fitting polynomial functions (e.g., the firstorder polynomial to adjust a linear trend) before calculation (Figures $1(\mathrm{c})$ and $1(\mathrm{~d})$ ), which aims at eliminating effects of 
nonstationarity due to, e.g., daily activities; the effects up the higher-order polynomials can be systematically examined.

\subsection{Considering Size and Location of Time Windows for Aggre-} gation of Physical Activity Data. One of the most important questions when examining the association between two (or more) constructs varying over time is how to address the time windows (i.e., location and size) that are used to aggregate each construct. The choice of the size of the time window may be important because it could have a significant impact on the robustness of the statistics and their temporal coincidence with the symptoms. Time windows can be chosen either by using theoretical rationale or by explorative examination. An example of the latter is described below.

One possible attempt is to systematically vary the size and location of the time windows to examine their effects [85]. For example, when the epoch length of physical activity obtained by accelerometer is $1-\mathrm{min}, 60-\mathrm{min}$ local mean or $S D$ of physical activity is computed from 60 data points, whereas 5-min local statistics are computed from 5 data points. Theoretically, the larger the size of the time window, the greater the stability and reliability of the estimates. However, the choice of larger time windows may obscure more transient fluctuations in the relations between physical activity and self-reported symptoms. Prior studies have used many different sizes of time windows to understand the associations between physical activity and affect states. There are many studies that have focused on very short time windows: 5-30 $\min [101,103,107,108]$, which may be useful to check transient associations among target variables or examine health benefits from an even short period of physical activity. One study systematically varied the size of time windows from 5 min (transient) to 2 hours (medium) with a 5-min time interval to test proper time windows predicting depressive mood [85]. Another study used medium (4 hours) time windows of physical activity to compare with affect states assessed every 4 hours [11]. The associations between physical activity and affect on a day level (i.e., relatively long time window) have also been examined $[109,110]$. Day-level time windows to aggregate physical activity may be used to examine overall associations with daily affect or event (e.g., sleep), but this examination is sometimes pragmatically made due to the limitation of sparse sampling (i.e., no observations within a day). The size of time windows largely depends upon the research question, but given the large freedom researchers typically have in selecting the size of time windows it is important that future research evaluates the reproducibility of the time-specific effects.

In addition, the choice of the location of the time window plays an important role in the investigation of causal associations, such as whether physical activity precedes or follows changes in momentary symptoms. There is scarcity of research on the bidirectional association between physical activity and momentary symptoms. Some studies showed that physical activity influences mental health benefits [108, 111], whereas others focused on how subjective symptoms predict subsequent physical activity $[102,108,112]$. However, it is a complicated domain and careful consideration of such a trade-off is important, although the optimal choice might be difficult to predict. Figures 1(a) and 1(b) are examples that show an examination of the temporal associations of depressive mood and local mean or detrended skewness of physical activity. Estimated values of the univariate multilevel model coefficient (i.e., slope) for the associations are shown in a colored matrix form consisting of 25 columns (different location) and 12 rows (different size). We considered 25 different locations $(-60,-55,-50, \ldots, 55,60 \mathrm{~min})$ and 12 different sizes $(120,110,100, \ldots, 20,10 \mathrm{~min})$. In total, we considered 300 combinations (25 locations $\times 12$ sizes) for local statistics of physical activity to examine the association with depressive mood assessed by EMA. More specifically, the top left cell in Figure 1(a) represents the model coefficient for the association between depressive mood (EMA) and local mean which calculated from the 10-min size of time window 60 min before EMA (i.e., from -60 to -50 min before EMA). Thus, the colored matrices generally show reduced mean or (detrended) positively skewed activity patterns preceded or occurred concurrently with a higher level of depressive mood rather than following. The false discovery rate with the $q$ value of .05 was used as the multiple comparison adjustment [113].

Although we discussed the size and location of time windows which are important when we explore the relationship with self-reported symptoms, the underlying mechanism of sustainability and causality alterations in the levels and patterns of physical activity with affect is uncertain. Further study using the data of high temporal resolution is necessary to clarify this question.

\section{Further Challenges}

Behavioral patterns characterized by reduced activity and intermittent bursts during low activity periods, as measured by accelerometer, are associated with EMA reports of worse depressive mood in healthy adolescents, older adults, undergraduates, office workers, and patients with MDD. This suggests that behavioral monitoring by the accelerometer may contribute to the identification of changes in subjective symptoms and improved management of these symptoms. While prior studies successfully provided a psychobehavioral measure based on accelerometer data, other types of timevarying changes in daily life should be examined to understand the associations between objective/subjective measures and health-related outcomes.

Many researchers and clinicians these days on the behavioral sciences and other scientific disciplines use mobile data collection incorporating information and communication technologies (ICTs), which enables a more refined understanding of psychiatric disorders including associations among various behavioral/physiological/biological measures. Furthermore, wearable devices (e.g., smartwatch) are increasingly popular to monitor health outcomes such as physical activity, sleep, and heart rate. The abundant information extracted from wearable devices is provided to numerous users often via smartphone applications and have great potential to elicit useful data for health outcomes in academic fields. Another challenge is how to use this information for improved monitoring, management, and intervention of health-related behaviors. For example, the concept 
of ecological momentary interventions (EMIs), in which real-time interventions are delivered to individuals during their everyday lives in natural settings, is a core elemental technology that is used for novel treatments of diseases including psychiatric disorders [114]. In addition, emerging electronic devices will make "context-sensitive prompting" possible, where questions are automatically triggered based on the subject's behavior, location, physiological states, past responses, and social interactions, which is considered useful for detecting early signs of psychiatric disorders and their pathological transitions $[106,115]$. However, actual realization and examination for these novel techniques are necessary in further studies.

\section{Conclusion}

In this paper, we introduced the multilevel modeling approach, which is useful for analyzing EMA data with observations hierarchically nested within individuals. Although new analytic challenges arise with addressing accelerometer data, it allows for nuanced characterizing of the temporal pattern of physical activity and its correlates. We exemplified different kinds of statistics (e.g., mean and skewness) of physical activity to extract activity patterns in various temporal time windows (i.e., size and location around EMA) which can be widely used according to research questions, but further studies using different types of statistics with a high temporal resolution are necessary to clarify these issues. Detailed SAS codes of multilevel models are shown in the Supplementary Materials.

\section{Conflicts of Interest}

The authors declare that there are no conflicts of interest regarding the publication of this paper.

\section{Acknowledgments}

This paper was supported by the National Sports Promotion Fund in accordance with the Sports Industry Technology Development Project of the Ministry of Culture, Sports and Tourism in 2017 (S072016032016). The authors would like to thank Drs. H. Shimura and Y. Yamamoto for their contribution to data collection for older adults which are used in Figure 1.

\section{Supplementary Materials}

The aim of the Supplementary Materials is to introduce SAS codes for multilevel modeling on the association between local statistics of physical activity based on accelerometer data and self-reported affect based on EMA. It consists of three parts: (a) Aggregating acceleration counts to various time windows (e.g., $60 \mathrm{~min}$ ). (b) Merging physical activity data with self-reported affect. (c) Reporting the code for multilevel modeling on the association between local statistics of physical activity and affect, as described in Section 2.1. (Supplementary Materials)

\section{References}

[1] S. Schiffman, A. A. Stone, and M. R. Hufford, "Ecological momentary assessment," Annual Review of Clinical Psychology, vol. 4, pp. 1-32, 2008.

[2] J. M. Hektner, J. A. Schmidt, and M. Csikszentmihalyi, Experience Sampling Method: Measuring The Quality of Everyday Life, SAGE Publications, Thousand Oaks, Calif, USA, 2007.

[3] T. J. Trull and U. Ebner-Priemer, "Ambulatory assessment," Annual Review of Clinical Psychology, vol. 9, pp. 151-176, 2013.

[4] A.-M. Vranceanu, L. C. Gallo, and L. M. Bogart, "Depressive symptoms and momentary affect: The role of social interaction variables," Depression and Anxiety, vol. 26, no. 5, pp. 464-470, 2009.

[5] J. Kim, H. Kikuchi, and Y. Yamamoto, "Systematic comparison between ecological momentary assessment and day reconstruction method for fatigue and mood states in healthy adults," British Journal of Health Psychology, vol. 18, no. 1, pp. 155-167, 2013.

[6] A. A. Stone, J. M. Smyth, T. Pickering, and J. Schwartz, "Daily mood variability: form of diurnal patterns and determinants of diurnal patterns," Journal of Applied Social Psychology, vol. 26, no. 14 , pp. 1286-1305, 1996.

[7] A. Steptoe, E. S. Leigh, and M. Kumari, "Positive affect and distressed affect over the day in older people," Psychology and Aging, vol. 26, no. 4, pp. 956-965, 2011.

[8] B. Farquharson, C. Bell, D. Johnston et al., "Nursing stress and patient care: Real-time investigation of the effect of nursing tasks and demands on psychological stress, physiological stress, and job performance: Study protocol," Journal of Advanced Nursing, vol. 69, no. 10, pp. 2327-2335, 2013.

[9] V. Thewissen, R. P. Bentall, T. Lecomte, J. van Os, and I. MyinGermeys, "Fluctuations in self-esteem and paranoia in the context of daily life," Journal of Abnormal Psychology, vol. 117, no. 1, pp. 143-153, 2008.

[10] R. A. Carels, O. M. Douglass, H. M. Cacciapaglia, and W. H. O'Brien, "An ecological momentary assessment of relapse crises in dieting," Journal of Consulting and Clinical Psychology, vol. 72, no. 2, pp. 341-348, 2004.

[11] G. F. Dunton, A. A. Atienza, C. M. Castro, and A. C. King, "Using ecological momentary assessment to examine antecedents and correlates of physical activity bouts in adults age 50+ years: a pilot study," Annals of Behavioral Medicine, vol. 38, no. 3, pp. 249-255, 2009.

[12] M. Wichers, F. Peeters, B. P. F. Rutten et al., "A time-lagged momentary assessment study on daily life physical activity and affect," Health Psychology, vol. 31, no. 2, pp. 135-144, 2012.

[13] S. Shiffman, M. H. Balabanis, C. J. Gwaltney et al., "Prediction of lapse from associations between smoking and situational antecedents assessed by ecological momentary assessment," Drug and Alcohol Dependence, vol. 91, no. 2-3, pp. 159-168, 2007.

[14] S. Chandra, D. Scharf, and S. Shiffman, "Within-day temporal patterns of smoking, withdrawal symptoms, and craving," Drug and Alcohol Dependence, vol. 117, no. 2-3, pp. 118-125, 2011.

[15] S. Shiffman and T. R. Kirchner, "Cigarette-by-cigarette satisfaction during ad libitum smoking," Journal of Abnormal Psychology, vol. 118, no. 2, pp. 348-359, 2009.

[16] M. Hillbrand and B. M. Waite, "The everyday experience of an institutionalized sex offender: An idiographic application of the experience sampling method," Archives of Sexual Behavior, vol. 23, no. 4, pp. 453-463, 1994. 
[17] A. Müller, J. E. Mitchell, R. D. Crosby et al., "Mood states preceding and following compulsive buying episodes: An ecological momentary assessment study," Psychiatry Research, vol. 200, no. 2-3, pp. 575-580, 2012.

[18] V. S. Helgeson, L. C. Lopez, and T. Kamarck, "Peer relationships and diabetes: retrospective and ecological momentary assessment approaches," Health Psychology, vol. 28, no. 3, pp. 273-282, 2009.

[19] T. Rutledge, E. Stucky, A. Dollarhide et al., "A real-time assessment of work stress in physicians and nurses," Health Psychology, vol. 28, no. 2, pp. 194-200, 2009.

[20] S. A. Mulvaney, R. L. Rothman, M. S. Dietrich et al., "Using mobile phones to measure adolescent diabetes adherence," Health Psychology, vol. 31, no. 1, pp. 43-50, 2012.

[21] D. E. McCarthy, T. M. Piasecki, D. L. Lawrence, D. E. Jorenby, S. Shiffman, and T. B. Baker, "Psychological mediators of bupropion sustained-release treatment for smoking cessation," Addiction, vol. 103, no. 9, pp. 1521-1533, 2008.

[22] H. Kikuchi, K. Yoshiuchi, Y. Yamamoto, G. Komaki, and A. Akabayashi, "Diurnal variation of tension-type headache intensity and exacerbation: An investigation using computerized ecological momentary assessment," BioPsychoSocial Medicine, vol. 6, article 8, 2012.

[23] T. Ritz, D. Rosenfield, S. DeWilde, and A. Steptoe, "Daily mood, shortness of breath, and lung function in asthma: Concurrent and prospective associations," Journal of Psychosomatic Research, vol. 69, no. 4, pp. 341-351, 2010.

[24] R. S. Everhart, J. M. Smyth, A. M. Santuzzi, and B. H. Fiese, "Validation of the asthma quality of life questionnaire with momentary assessments of symptoms and functional limitations in patient daily life," Respiratory Care, vol. 55, no. 4, pp. 427-432, 2010.

[25] G. D'Amato and L. Cecchi, "Effects of climate change on environmental factors in respiratory allergic diseases," Clinical \& Experimental Allergy, vol. 38, no. 8, pp. 1264-1274, 2008.

[26] M. Saito, H. Kumano, K. Yoshiuchi et al., "Symptom profile of multiple chemical sensitivity in actual life," Psychosomatic Medicine, vol. 67, no. 2, pp. 318-325, 2005.

[27] J. A. Henry, G. Galvez, M. B. Turbin, E. J. Thielman, G. P. McMillan, and J. A. Istvan, "Pilot study to evaluate ecological momentary assessment of tinnitus," Ear and Hearing, vol. 33, no. 2, pp. 179-290, 2012.

[28] R. M. Schuster, R. J. Mermelstein, and D. Hedeker, "Ecological momentary assessment of working memory under conditions of simultaneous marijuana and tobacco use," Addiction, vol. 111, no. 8, pp. 1466-1476, 2016.

[29] J. A. Cramer, R. H. Mattson, M. L. Prevey, R. D. Scheyer, and V. L. Ouellette, "How often is medication taken as prescribed?: a novel assessment technique," Journal of the American Medical Association, vol. 261, no. 22, pp. 3273-3277, 1989.

[30] L. van Wel, A. Huss, P. Bachmann et al., "Context-sensitive ecological momentary assessments; integrating real-time exposure measurements, data-analytics and health assessment using a smartphone application," Environment International, vol. 103, pp. 8-12, 2017.

[31] J. R. Turner, M. M. Ward, M. D. Gellman, D. W. Johnston, K. C. Light, and L. J. P. Van Doornen, "The relationship between laboratory and ambulatory cardiovascular activity: current evidence and future directions," Annals of Behavioral Medicine, vol. 16, pp. 12-23, 1994.

[32] B. A. Teicher, S. A. Holden, N. P. Dupuis et al., "Potentiation of cytotoxic therapies by TNP-470 and minocycline in mice bearing EMT-6 mammary carcinoma," Breast Cancer Research and Treatment, vol. 36, no. 2, pp. 227-236, 1995.

[33] M. H. Teicher, C. A. Glod, E. Magnus et al., "Circadian restactivity disturbances in seasonal affective disorder," Archives of General Psychiatry, vol. 54, no. 2, pp. 124-130, 1997.

[34] C. Burton, B. McKinstry, A. Szentagotai Tătar, A. SerranoBlanco, C. Pagliari, and M. Wolters, "Activity monitoring in patients with depression: a systematic review," Journal of Affective Disorders, vol. 145, no. 1, pp. 21-28, 2013.

[35] P. Indic, G. Murray, C. Maggini et al., "Multi-scale motility amplitude associated with suicidal thoughts in major depression," PLoS ONE, vol. 7, no. 6, Article ID e38761, 2012.

[36] S. Walther, S. Hügli, O. Höfle et al., "Frontal white matter integrity is related to psychomotor retardation in major depression," Neurobiology of Disease, vol. 47, no. 1, pp. 13-19, 2012.

[37] A. C. Volkers, J. H. M. Tulen, W. W. Van Den Broek, J. A. Bruijn, J. Passchier, and L. Pepplinkhuizen, "Motor activity and autonomic cardiac functioning in major depressive disorder," Journal of Affective Disorders, vol. 76, no. 1-3, pp. 23-30, 2003.

[38] J. O. Berle, E. R. Hauge, K. J. Oedegaard, F. Holsten, and O. B. Fasmer, "Actigraphic registration of motor activity reveals a more structured behavioural pattern in schizophrenia than in major depression," BMC Research Notes, vol. 3, article 149, 2010.

[39] T. F. Robles, V. Shetty, C. M. Zigler et al., "The feasibility of ambulatory biosensor measurement of salivary alpha amylase: relationships with self-reported and naturalistic psychological stress," Biological Psychology, vol. 86, no. 1, pp. 50-56, 2011.

[40] M. Van Eck, H. Berkhof, N. Nicolson, and J. Sulon, "The effects of perceived stress, traits, mood states, and stressful daily events on salivary cortisol," Psychosomatic Medicine, vol. 58, no. 5, pp. 447-458, 1996.

[41] J. Smyth, M. C. Ockenfels, L. Porter, C. Kirschbaum, D. H. Hellhammer, and A. A. Stone, "Stressors and mood measured on a momentary basis are associated with salivary cortisol secretion," Psychoneuroendocrinology, vol. 23, no. 4, pp. 353370, 1998.

[42] A. Steptoe, E. Leigh Gibson, M. Hamer, and J. Wardle, "Neuroendocrine and cardiovascular correlates of positive affect measured by ecological momentary assessment and by questionnaire," Psychoneuroendocrinology, vol. 32, no. 1, pp. 56-64, 2007.

[43] E. Boland, T. Monsod, M. Delucia, C. A. Brandt, S. Fernando, and W. V. Tamborlane, "Limitations of conventional methods of self-monitoring of blood glucose: lessons learned from 3 days of continuous glucose sensing in pediatric patients with type 1 diabetes," Diabetes Care, vol. 24, no. 11, pp. 1858-1862, 2001.

[44] T. R. Leffingwell, N. J. Cooney, J. G. Murphy et al., "Continuous objective monitoring of alcohol use: twenty-first century measurement using transdermal sensors," Alcoholism: Clinical and Experimental Research, vol. 37, no. 1, pp. 16-22, 2013.

[45] M. J. Sorbi, S. B. Mak, J. H. Houtveen, A. M. Kleiboer, and L. J. P. Van Doornen, "Mobile web-based monitoring and coaching: Feasibility in chronic migraine," Journal of Medical Internet Research, vol. 9, no. 5, p. e38, 2007.

[46] S. Munsch, A. H. Meyer, N. Milenkovic, B. Schlup, J. Margraf, and F. H. Wilhelm, "Ecological momentary assessment to evaluate cognitive-behavioral treatment for binge eating disorder," International Journal of Eating Disorders, vol. 42, no. 7, pp. 648657, 2009.

[47] G. Jónasson, K.-H. Carlsen, A. Sødal, C. Jonasson, and P. Mowinckel, "Patient compliance in a clinical trial with inhaled 
budesonide in children with mild asthma," European Respiratory Journal, vol. 14, no. 1, pp. 150-154, 1999.

[48] K. E. MacDonell, S. Naar-King, D. A. Murphy, J. T. Parsons, and H. Huszti, "Situational temptation for HIV medication adherence in high-risk youth," AIDS Patient Care and STDs, vol. 25, no. 1, pp. 47-52, 2011.

[49] K. Basen-Engquist, C. L. Carmack, Y. Li et al., "Social-cognitive theory predictors of exercise behavior in endometrial cancer survivors," Health Psychology, vol. 32, no. 11, pp. 1137-1148, 2013.

[50] B. C. Focht, V. Ewing, L. Guavin, and W. J. Rejeski, "The unique and transient impact of acute exercise on pain perception in older, overweight, or obese adults with knee osteoarthritis," Annals of Behavioral Medicine, vol. 24, no. 3, pp. 201-210, 2002.

[51] D. H. Epstein, G. F. Marrone, S. J. Heishman, J. Schmittner, and K. L. Preston, "Tobacco, cocaine, and heroin: Craving and use during daily life," Addictive Behaviors, vol. 35, no. 4, pp. 318-324, 2010.

[52] M. J. Freedman, K. M. Lester, C. McNamara, J. B. Milby, and J. E. Schumacher, "Cell phones for ecological momentary assessment with cocaine-addicted homeless patients in treatment," Journal of Substance Abuse Treatment, vol. 30, no. 2, pp. 105-111, 2006.

[53] S. R. Weinland, C. B. Morris, Y. Hu, J. Leserman, S. I. Bangdiwala, and D. A. Drossman, "Characterization of episodes of irritable bowel syndrome using ecological momentary assessment," American Journal of Gastroenterology, vol. 106, no. 10, pp. 18131820, 2011.

[54] P. Jern, A. Gunst, F. Sandqvist, N. K. Sandnabba, and P. Santtila, "Using ecological momentary assessment to investigate associations between ejaculatory latency and control in partnered and non-partnered sexual activities," The Journal of Sex Research, vol. 48, no. 4, pp. 316-324, 2011.

[55] C. Zunker, C. B. Peterson, R. D. Crosby et al., "Ecological momentary assessment of bulimia nervosa: Does dietary restriction predict binge eating?" Behaviour Research and Therapy, vol. 49, no. 10, pp. 714-717, 2011.

[56] C. Burd, J. E. Mitchell, R. D. Crosby et al., "An assessment of daily food intake in participants with anorexia nervosa in the natural environment," International Journal of Eating Disorders, vol. 42, no. 4, pp. 371-374, 2009.

[57] P. J. Rosen, J. N. Epstein, and G. Van Orden, "I know it when I quantify it: Ecological momentary assessment and recurrence quantification analysis of emotion dysregulation in children with ADHD," ADHD Attention Deficit and Hyperactivity Disorders, vol. 5, no. 3, pp. 283-294, 2013.

[58] L. E. Knouse, J. T. Mitchell, L. H. Brown et al., “The expression of adult ADHD symptoms in daily life: An application of experience sampling methodology," Journal of Attention Disorders, vol. 11, no. 6, pp. 652-663, 2008.

[59] U. W. Ebner-Priemer, J. Kuo, W. Schlotz et al., "Distress and affective dysregulation in patients with borderline personality disorder: A psychophysiological ambulatory monitoring study," The Journal of Nervous and Mental Disease, vol. 196, no. 4, pp. 314-320, 2008.

[60] P. Z. Tan, E. E. Forbes, R. E. Dahl et al., "Emotional reactivity and regulation in anxious and nonanxious youth: A cellphone ecological momentary assessment study," Journal of Child Psychology and Psychiatry and Allied Disciplines, vol. 53, no. 2, pp. 197-206, 2012.

[61] M. C. Pfaltz, T. Michael, P. Grossman, J. Margraf, and F. H. Wilhelm, "Instability of physical anxiety symptoms in daily life of patients with panic disorder and patients with posttraumatic stress disorder," Journal of Anxiety Disorders, vol. 24, no. 7, pp. 792-798, 2010.

[62] S. Helbig-Lang, T. Lang, F. Petermann, and J. Hoyer, "Anticipatory anxiety as a function of panic attacks and panic-related selfefficacy: An ambulatory assessment study in panic disorder," Behavioural and Cognitive Psychotherapy, vol. 40, no. 5, pp. 590604, 2012.

[63] E. E. Forbes, A. R. Hariri, S. L. Martin et al., "Altered striatal activation predicting real-world positive affect in adolescent major depressive disorder," The American Journal of Psychiatry, vol. 166, no. 1, pp. 64-73, 2009.

[64] L. M. Bylsma, A. Taylor-Clift, and J. Rottenberg, "Emotional reactivity to daily events in major and minor depression," Journal of Abnormal Psychology, vol. 120, no. 1, pp. 155-167, 2011.

[65] F. Peeters, N. A. Nicolson, J. Berkhof, P. Delespaul, and M. De Vries, "Effects of daily events on mood states in major depressive disorder," Journal of Abnormal Psychology, vol. 112, no. 2, pp. 203-211, 2003.

[66] R. Havermans, N. A. Nicolson, J. Berkhof, and M. W. deVries, "Mood reactivity to daily events in patients with remitted bipolar disorder," Psychiatry Research, vol. 179, no. 1, pp. 47-52, 2010.

[67] D. Kimhy, P. Delespaul, C. Corcoran, H. Ahn, S. Yale, and D. Malaspina, "Computerized experience sampling method (ESMc): Assessing feasibility and validity among individuals with schizophrenia," Journal of Psychiatric Research, vol. 40, no. 3, pp. 221-230, 2006.

[68] E. Granholm, D. Ben-Zeev, D. Fulford, and J. Swendsen, "Ecological Momentary Assessment of social functioning in schizophrenia: Impact of performance appraisals and affect on social interactions," Schizophrenia Research, vol. 145, no. 1-3, pp. 120-124, 2013.

[69] E. Granholm, C. Loh, and J. Swendsen, "Feasibility and validity of computerized ecological momentary assessment in schizophrenia," Schizophrenia Bulletin, vol. 34, no. 3, pp. 507514, 2008.

[70] S. J. Wilson, L. M. Martire, and M. J. Sliwinski, “Daily spousal responsiveness predicts longer-term trajectories of patients' physical function," Psychological Science, vol. 28, no. 6, pp. 786797, 2017.

[71] T. W. Kamarck, J. E. Schwartz, S. Shiffman, M. F. Muldoon, K. Sutton-Tyrrell, and D. L. Janicki, "Psychosocial stress and cardiovascular risk: what is the role of daily experience?" Journal of Personality, vol. 73, no. 6, pp. 1749-1774, 2005.

[72] T. W. Kamarck, S. M. Shiffman, L. Smithline et al., "Effects of task strain, social conflict, and emotional activation on ambulatory cardiovascular activity: Daily life consequences of recurring stress in a multiethnic adult sample," Health Psychology, vol. 17, no. 1, pp. 17-29, 1998.

[73] T. W. Smith, W. Birmingham, and B. N. Uchino, "Evaluative threat and ambulatory blood pressure: Cardiovascular effects of social stress in daily experience," Health Psychology, vol. 31, no. 6, pp. 763-766, 2012.

[74] P. Grossman, G. Deuring, S. N. Garland, T. S. Campbell, and L. E. Carlson, "Patterns of objective physical functioning and perception of mood and fatigue in posttreatment breast cancer patients and healthy controls: An ambulatory psychophysiological investigation," Psychosomatic Medicine, vol. 70, no. 7, pp. 819-828, 2008. 
[75] V. Bitsika, C. F. Sharpley, N. M. Andronicos, and L. L. Agnew, "Hypothalamus-pituitary-adrenal axis daily fluctuation, anxiety and age interact to predict cortisol concentrations in boys with an autism spectrum disorder," Physiology \& Behavior, vol. 138, pp. 200-207, 2015.

[76] J. M. Lavender, K. P. De Young, S. A. Wonderlich et al., "Daily patterns of anxiety in anorexia nervosa: Associations with eating disorder behaviors in the natural environment," Journal of Abnormal Psychology, vol. 122, no. 3, pp. 672-683, 2013.

[77] R. D. Crosby, S. A. Wonderlich, S. G. Engel, H. Simonich, J. Smyth, and J. E. Mitchell, "Daily mood patterns and bulimic behaviors in the natural environment," Behaviour Research and Therapy, vol. 47, no. 3, pp. 181-188, 2009.

[78] M. Muraven, R. L. Collins, S. Shiffman, and J. A. Paty, "Daily fluctuations in self-control demands and alcohol intake," Psychology of Addictive Behaviors, vol. 19, no. 2, pp. 140-147, 2005.

[79] S. Jahng, M. B. Solhan, R. L. Tomko, P. K. Wood, T. M. Piasecki, and T. J. Trull, "Affect and alcohol use: An ecological momentary assessment study of outpatients with borderline personality disorder," Journal of Abnormal Psychology, vol. 120, no. 3, pp. 572-584, 2011.

[80] American Psychiatric Association, Diagnostic and Statistical Manual of Mental Disorders, American Psychiatric Press, Washington, Wash, USA, 4th edition, 2000.

[81] T. Nakamura, K. Kiyono, K. Yoshiuchi, R. Nakahara, Z. R. Struzik, and Y. Yamamoto, "Universal scaling law in human behavioral organization," Physical Review Letters, vol. 99, no. 13, Article ID 138103, 2007.

[82] T. Nakamura, T. Takumi, A. Takano et al., "Of mice and men Universality and breakdown of behavioral organization," PLoS ONE, vol. 3, no. 4, Article ID e2050, 2008.

[83] W. Sano, T. Nakamura, K. Yoshiuchi et al., "Enhanced persistency of resting and active periods of locomotor activity in schizophrenia," PLoS ONE, vol. 7, no. 8, Article ID e43539, 2012.

[84] T. Nakamura, K. Kiyono, H. Wendt, P. Abry, and Y. Yamamoto, "Multiscale analysis of intensive longitudinal biomedical signals and its clinical applications," Proceedings of the IEEE, vol. 104, no. 2, pp. 242-261, 2016.

[85] J. Kim, T. Nakamura, H. Kikuchi, T. Sasaki, and Y. Yamamoto, "Co-variation of depressive mood and locomotor dynamics evaluated by ecological momentary assessment in healthy humans," PLoS ONE, vol. 8, no. 9, Article ID e74979, 2013.

[86] J. Kim, T. Nakamura, H. Kikuchi, K. Yoshiuchi, T. Sasaki, and Y. Yamamoto, "Covariation of depressive mood and spontaneous physical activity in major depressive disorder: toward continuous monitoring of depressive mood," IEEE Journal of Biomedical and Health Informatics, vol. 19, no. 4, pp. 1347-1355, 2015.

[87] J. Kim, F. Togo, H. Shimura, A. Yasunaga, T. Nakamura et al., "Associations between spontaneous physical activity and mood states in older adults: an ambulatory assessment approach in daily life," in Proceedings of the The 4th Biennial Conference of Ambulatory Assessment, State College, Pa, USA, 2015.

[88] B. von Haaren, S. N. Loeffler, S. Haertel et al., "Characteristics of the activity-affect association in inactive people: an ambulatory assessment study in daily life," Frontiers in Psychology, vol. 4, article 163, 2013.

[89] J. Mata, R. J. Thompson, S. M. Jaeggi, M. Buschkuehl, J. Jonides, and I. H. Gotlib, "Walk on the bright side: Physical activity and affect in major depressive disorder," Journal of Abnormal Psychology, vol. 121, no. 2, pp. 297-308, 2012.
[90] J. E. Schwartz and A. A. Stone, "Strategies for analyzing ecological momentary assessment data," Health Psychology, vol. 17, no. 1, pp. 6-16, 1998.

[91] J. J. Hox and J. K. Roberts, Handbook of Advanced Multilevel Analysis, Routledge, New York, NY, USA, 2011.

[92] J. D. Singer and J. B. Willett, Applied Longitudinal Data Analysis: Modeling Change and Event Occurrence, Oxford University Press, Oxford, UK, 2003.

[93] C. K. Enders and D. Tofighi, "Centering predictor variables in cross-sectional multilevel models: a new look at an old issue," Psychological Methods, vol. 12, no. 2, pp. 121-138, 2007.

[94] S. Ancoli-Israel, R. Cole, C. Alessi, M. Chambers, W. Moorcroft, and C. P. Pollak, "The role of actigraphy in the study of sleep and circadian rhythms," SLEEP, vol. 26, no. 3, pp. 342-392, 2003.

[95] R. P. Troiano, J. J. McClain, R. J. Brychta, and K. Y. Chen, "Evolution of accelerometer methods for physical activity research," British Journal of Sports Medicine, vol. 48, pp. 1019-1023, 2014.

[96] J. A. Banda, K. F. Haydel, T. Davila et al., "Effects of varying epoch lengths, wear time algorithms, and activity cut-points on estimates of child sedentary behavior and physical activity from accelerometer data," PLoS ONE, vol. 11, no. 3, Article ID e0150534, 2016.

[97] M. S. Tremblay, S. Aubert, J. D. Barnes et al., "Sedentary Behavior Research Network (SBRN) - Terminology Consensus Project process and outcome," International Journal of Behavioral Nutrition and Physical Activity, vol. 14, no. 1, 2017.

[98] N. Owen, G. N. Healy, C. E. Matthews, and D. W. Dunstan, "Too much sitting: the population health science of sedentary behavior," Exercise and Sport Sciences Reviews, vol. 38, no. 3, pp. 105-113, 2010.

[99] E. G. Wilmot, C. L. Edwardson, and F. A. Achana, "Sedentary time in adults and the association with diabetes, cardiovascular disease and death: systematic review and meta-analysis," Diabetologia, vol. 55, no. 11, pp. 2895-2905, 2012, Erratum in: Diabetologia, vol. 56, no. 4, pp. 942-943, 2013.

[100] A. A. Thorp, N. Owen, M. Neuhaus, and D. W. Dunstan, "Sedentary behaviors and subsequent health outcomes in adults: a systematic review of longitudinal studies, 1996-2011," American Journal of Preventive Medicine, vol. 41, no. 2, pp. 207-215, 2011.

[101] Y. Liao, C.-P. Chou, J. Huh, A. Leventhal, and G. Dunton, "Examining acute bi-directional relationships between affect, physical feeling states, and physical activity in free-living situations using electronic ecological momentary assessment," Journal of Behavioral Medicine, vol. 40, no. 3, pp. 445-457, 2017.

[102] C. Y. Niermann, C. Herrmann, B. von Haaren, D. van Kann, and A. Woll, "Affect and subsequent physical activity: an ambulatory assessment study examining the affect-activity association in a real-life context," Frontiers in Psychology, vol. 7, article 677, 2016.

[103] G. F. Dunton, J. Huh, A. M. Leventhal et al., "Momentary assessment of affect, physical feeling states, and physical activity in children," Health Psychology, vol. 33, no. 3, pp. 255-263, 2014.

[104] B. Castaing, Y. Gagne, and E. J. Hopfinger, "Velocity probability density functions of high Reynolds number turbulence," Physica D: Nonlinear Phenomena, vol. 46, no. 2, pp. 177-200, 1990.

[105] K. Kiyono, "Log-amplitude statistics of intermittent and nonGaussian time series," Physical Review E: Statistical, Nonlinear, and Soft Matter Physics, vol. 79, no. 3, 2009.

[106] A. A. Stone et al., The Science of Real-Time Data Capture: SelfReports in Health Research, Oxford University Press, Oxford, UK, 2007. 
[107] T. Bossmann, M. Kanning, S. Koudela-Hamila, S. Hey, and U. Ebner-Priemer, "The association between short periods of everyday life activities and affective states: a replication study using ambulatory assessment," Frontiers in Psychology, vol. 4, article 102, 2013.

[108] A. Schwerdtfeger, R. Eberhardt, A. Chmitorz, and E. Schaller, "Momentary affect predicts bodily movement in daily life: An ambulatory monitoring study," Journal of Sport \& Exercise Psychology, vol. 32, no. 5, pp. 674-693, 2010.

[109] D. Aggio, K. Wallace, N. Boreham, A. Shankar, A. Steptoe, and M. Hamer, "Objectively measured daily physical activity and postural changes as related to positive and negative affect using ambulatory monitoring assessments," Psychosomatic Medicine, vol. 79, no. 7, pp. 792-797, 2017.

[110] A. Schöndube, M. Kanning, and R. Fuchs, "The bidirectional effect between momentary affective states and exercise duration on a day level," Frontiers in Psychology, vol. 7, article 1414, 2016.

[111] M. Hamer, N. Coombs, and E. Stamatakis, "Associations between objectively assessed and self-reported sedentary time with mental health in adults: An analysis of data from the health survey for England," BMJ Open, vol. 4, no. 3, Article ID e004580, 2014.

[112] Y. Liao, E. T. Shonkoff, and G. F. Dunton, "The acute relationships between affect, physical feeling states, and physical activity in daily life: A review of current evidence," Frontiers in Psychology, vol. 6, article 1975, 2015.

[113] Y. Benjamini and D. Yekutieli, "The control of the false discovery rate in multiple testing under dependency," The Annals of Statistics, vol. 29, no. 4, pp. 1165-1188, 2001.

[114] K. E. Heron and J. M. Smyth, "Ecological momentary interventions: incorporating mobile technology into psychosocial and health behaviour treatments," British Journal of Health Psychology, vol. 15, pp. 1-39, 2010.

[115] Z. R. Struzik, K. Yoshiuchi, M. Sone et al., “"Mobile nurse” platform for ubiquitous medicine," Methods of Information in Medicine, vol. 46, no. 2, pp. 130-134, 2007. 


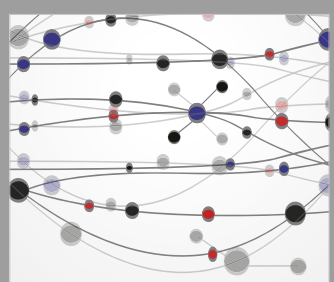

The Scientific World Journal
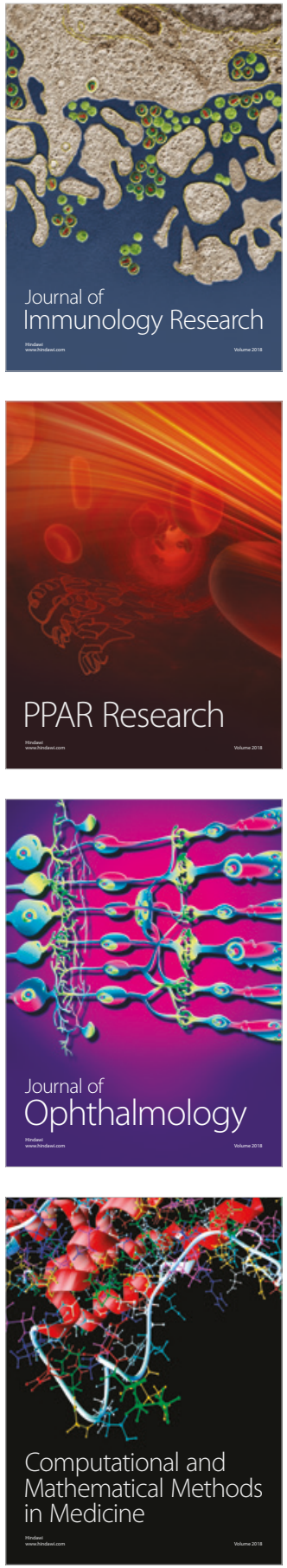

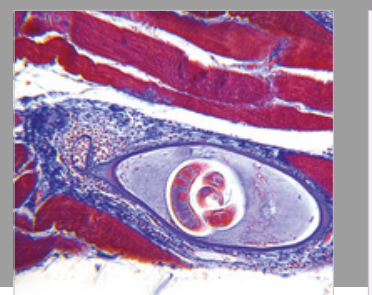

Gastroenterology Research and Practice

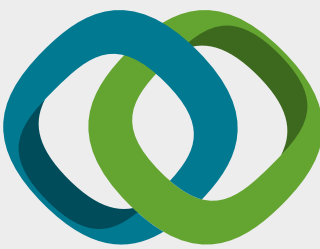

\section{Hindawi}

Submit your manuscripts at

www.hindawi.com
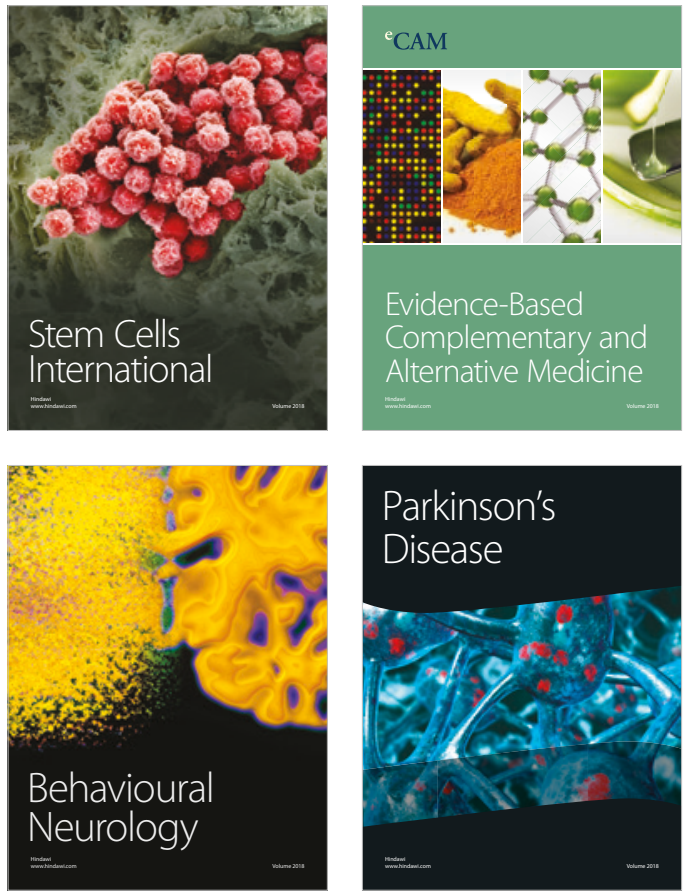

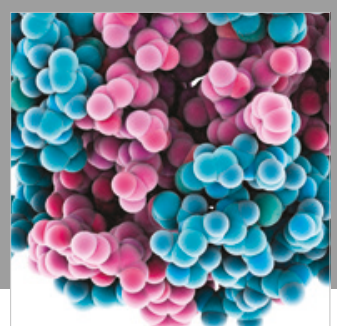

ournal of

Diabetes Research

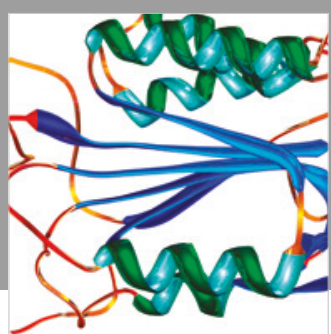

Disease Markers
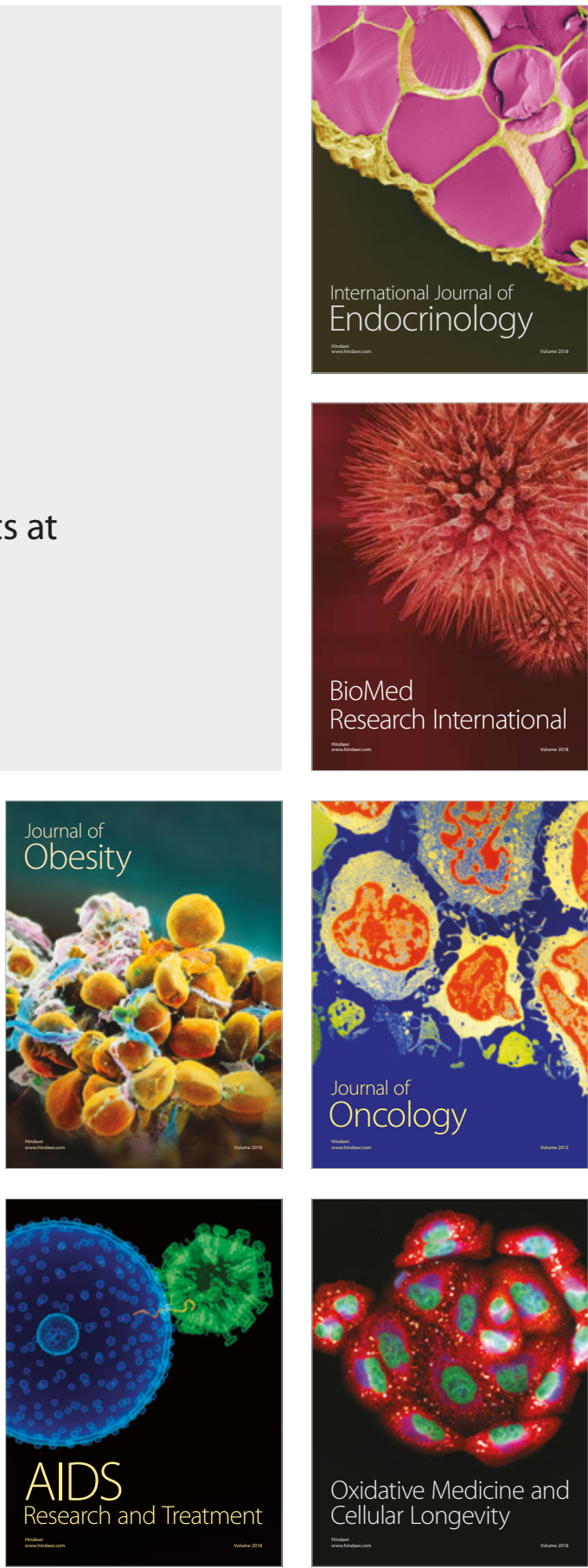